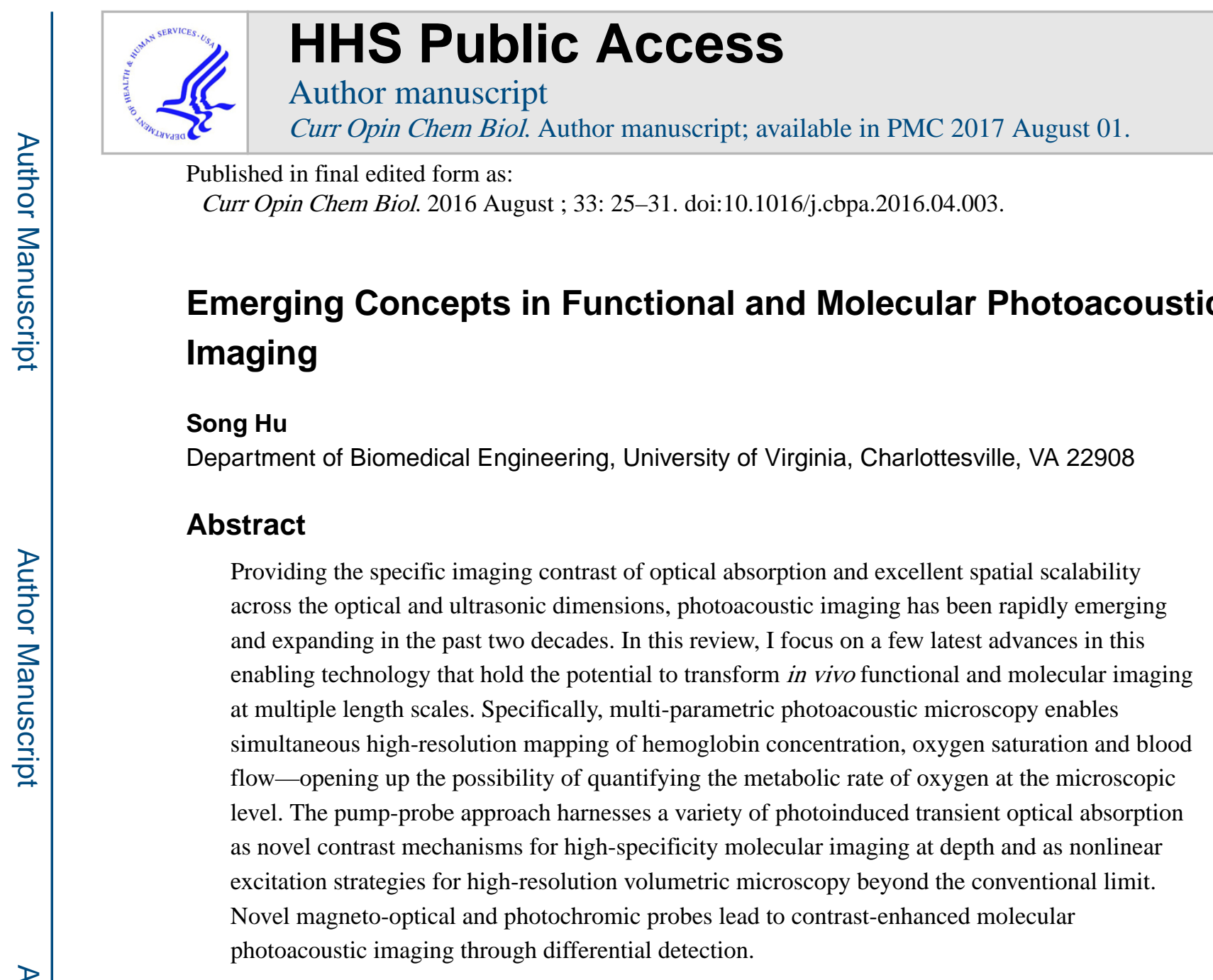

\title{
Introduction
}

Photoacoustic imaging, presenting an elegant way to combine optics and ultrasound, is among the most rapidly growing technologies in biomedicine [1,2]. In this emerging modality, endogenous or exogenous chromophores in the biological tissue are usually excited by nanosecond laser pulses. Part of the absorbed photon energy induces transient heating of the tissue through nonradiative relaxation of the excited states. The subsequent thermoelastic expansion converts the tiny temperature rise (typically at the level of milliKelvin) into ultrasonic emission, which can be captured by an acoustic detector to form a high-resolution image of the chromophore distribution.

The conversion of optical excitation to acoustic emission confers two distinct advantages to photoacoustic imaging. First, it provides the specific contrast of optical absorption, which ideally complements that of fluorescence imaging - the current technology of choice for optical molecular imaging in vivo. Indeed, photoacoustic imaging has enabled highsensitivity detection of a variety of endogenous chromophores that are intrinsically weakly fluorescent and difficult to label with exogenous fluorophores-including but not limited to cytochrome [3], hemoglobin [4], myoglobin [5], melanin [6], water [7] and lipid [8]. Labelfree photoacoustic imaging of these fluorescently "dark" chromophores has opened up new

Correspondence: songhu@virginia.edu. 
avenues for basic and translational research in brain ischemia [9], cancer [10,11] and atherosclerosis [12]. Second, the hybrid of light and sound allows seamless scalability of the spatial resolution and penetration depth of photoacoustic imaging across the microscopic (i.e., micrometer resolution at the depth of sub-millimeter), mesoscopic (i.e., tens of micrometer resolution at the depth of 1-10 mm) and macroscopic (i.e., 100-400 $\mu \mathrm{m}$ resolution at the depth of several centimeters) scales with an excellent depth-to-resolution ratio of 200 [2,13]. This scalability is not yet achievable with fluorescence-based molecular imaging. Although capable of providing microscopic resolution within the optical diffusion limit (i.e., $\sim 1 \mathrm{~mm}$ in biological tissues), fluorescence imaging has a poor depth-to-resolution ratio of $\sim 3$ in the optical diffusive regime [14]. With advances in optically absorbing probes, the photoacoustic effect provides an ideal mechanism for high-resolution molecular imaging beyond the diffusion limit.

In recent years, intensive research efforts have been made to unleash the full potential of photoacoustic imaging of both endogenous nonfluorescent chromophores and exogenous molecular probes. In this short review, I focus on only a few latest and potentially transformative advances in functional and molecular photoacoustic imaging_including (1) multi-parametric quantification of hemodynamics with the contrast of endogenous hemoglobin and its potential in oxygen-metabolic imaging at the microscopic level; (2) pump-probe interrogation of photoinduced spatiotemporal changes in the generation of photoacoustic signals and its applications in both high-specificity molecular imaging at depth and high-resolution volumetric imaging beyond the conventional limit; (3) photochromic and magneto-optical probes for high-contrast molecular photoacoustic imaging based on differential detection. Comprehensive reviews on functional and molecular photoacoustic imaging can be found in $[15,16]$.

\section{Multi-parametric photoacoustic microscopy}

Among the multiple endogenous chromophores that can be photoacoustically detected, hemoglobin is of particular importance. As the primary carrier of oxygen in the blood circulation, hemoglobin encodes vital metabolic information. Dysfunctions in oxygen metabolism has been increasingly recognized as a critical factor in the origination and progression of multiple life-threatening diseases, including cancer, ischemic stroke and neurodegeneration [17]. In vivo high-resolution imaging of the metabolic rate of oxygen $\left(\mathrm{MRO}_{2}\right)$ in mice-a species with well-established disease models and genetic manipulations -is crucial for understanding metabolism-related pathogenic mechanisms and formulating new therapeutic strategies.

Capable of measuring the concentration of hemoglobin $\left(\mathrm{C}_{\mathrm{Hb}}\right)$, oxygen saturation of hemoglobin $\left(\mathrm{sO}_{2}\right)$ and blood flow - the three parameters required for $\mathrm{MRO}_{2}$ quantification, photoacoustic microscopy (PAM) is ideally suited for oxygen-metabolic imaging in vivo. Indeed, PAM of $\mathrm{MRO}_{2}$ has been demonstrated first in the tumor-bearing mouse ear [18] and later in the electrically stimulated mouse brain [19] by measuring $\mathrm{C}_{\mathrm{Hb}}, \mathrm{sO}_{2}$ and blood flow at selected locations in the feeding arteries and draining veins of the region of interest. Although encouraging, this method has fundamental limitations. First, it can only be applied to the regions that have well-defined feeding arteries and draining veins. Moreover, the 
volumetric flow rates in the feeding and draining vessels must be equal (i.e., a closed circulation). Even if applicable, this method only quantifies the total $\mathrm{MRO}_{2}$ over the entire region. The lack of the ability to resolve $\mathrm{MRO}_{2}$ at the microscopic level limits its utility in studying the metabolic heterogeneity in cancer and ischemia.

A recent innovation, multi-parametric PAM, overcomes all these limitations by enabling simultaneous imaging of $\mathrm{C}_{\mathrm{Hb}}, \mathrm{sO}_{2}$ and blood flow at the microvascular level [20,21]. As shown in Fig. 1A, statistical, spectroscopic and correlation analysis of successively acquired dual-wavelength (i.e., 532 and $558 \mathrm{~nm}$ ) A- line pairs allows quantification of $\mathrm{C}_{\mathrm{Hb}}, \mathrm{sO}_{2}$ and blood flow at the same spatiotemporal scale. Specifically at $532 \mathrm{~nm}$, where the optical absorption of oxy- and deoxy-hemoglobin $\left(\mathrm{HbO}_{2}\right.$ and $\left.\mathrm{HbR}\right)$ are identical, $\mathrm{PAM}$ is insensitive to $\mathrm{SO}_{2}$. Fluctuations in the PAM signal acquired at this wavelength encode both the Brownian motion and the flow of red blood cells (RBCs) [22]. The Brownian motioninduced statistical fluctuation in the amplitude of these A-lines depends on the RBC count within the detection volume of PAM but not the blood flow, and thus can be used to derive $\mathrm{C}_{\mathrm{Hb}}$ in absolute values [23]. In parallel, the speed of blood flow can be quantified by examining the cross-correlation of the same set of A-lines [20]. The decay rate of the correlation reveals the dwell time of RBCs in the detection volume. The faster the decorrelation, the higher the flow speed. Taking advantage of bi-directional raster scan, PAM can further determine the direction of blood flow. In addition, by comparing the readouts at both wavelengths, $\mathrm{PAM}$ can assess the proportions of $\mathrm{HbO}_{2}$ and $\mathrm{HbR}$ in $\mathrm{C}_{\mathrm{Hb}}$, from which $\mathrm{sO}_{2}$ can be derived. With the quantitative analyses, multi-parametric PAM has demonstrated simultaneous high-resolution imaging of $\mathrm{C}_{\mathrm{Hb}}, \mathrm{sO}_{2}$ and blood flow velocity (i.e., both the speed and direction) in vivo (Fig. 1B-1D) [21]. Future development of complementary algorithms to extend these hemodynamic parameters from the microvascular level to the tissue level will ultimately enable $\mathrm{MRO}_{2}$ quantification at the microscopic level by using the Fick's law.

\section{Pump-probe photoacoustic imaging}

Pump-probe imaging of transient optical absorption is an emerging field in nonlinear microscopy [24,25]. Recently, this concept has been adopted by photoacoustic imaging for enriching the molecular contrast and refining the spatial resolution. This innovation has expanded the envelope of photoacoustic imaging by enabling lifetime and fluorophore imaging at depth and high-resolution volumetric imaging beyond the conventional limit. Three main mechanisms of current pump-probe photoacoustic imaging-excited-state absorption, stimulated emission and ground-state depletion—are introduced here.

\section{Lifetime imaging based on excited-state absorption}

In the case of excited-state absorption (top row of Fig. 2A), the pump laser pulse excites the molecule to be imaged from the ground state $\left(S_{0}\right)$ to an excited state $\left(S_{1}\right)$. The molecule can either decay back to $S_{0}$ via radiative or nonradiative relaxation or be promoted to a higher state $\left(S_{2}\right)$ by absorbing a photon from the probe pulse. Thus, populating $S_{1}$ with the pump excitation increases the molecule's absorptivity of the probe light. The differential optical absorption at the probe wavelength in the presence and absence of the pump encodes the 
energy structure of the molecule and can be utilized as a contrast mechanism for molecular photoacoustic imaging with high specificity. More excitingly, dynamic adjustments of the delay between the pump and probe pulses allow photoacoustic imaging of the depopulation rate of the excited state, from which its lifetime can be estimated.

Recently, excited-state absorption-based photoacoustic lifetime imaging has been combined with oxygen-sensitive dyes (e.g., methylene blue) to measure the partial pressure of oxygen $\left(\mathrm{pO}_{2}\right)$ at depths up to $12 \mathrm{~mm}$ [26]. Since the excited state of the dye molecule can be dynamically quenched through its collision with the surrounding oxygen molecules, photoacoustic imaging of its excited-state lifetime provides a quantitative measure of $\mathrm{pO}_{2}$ (Fig. 2B) [27]. Future integration of the lifetime measurement of $\mathrm{pO}_{2}$ and the spectroscopic measurement of $\mathrm{sO}_{2}$ will equip photoacoustic imaging with the unique capability of comprehensively characterizing the oxygen dynamics in vivo.

\section{Fluorophore imaging based on stimulated emission}

In contrast to excited-state absorption, which induces transient optical absorption at the probe wavelength, stimulated emission can be utilized to modulate the transient optical absorption at the pump wavelength. This mechanism has been applied to extend the scope of photoacoustic imaging from chromophores to fluorophores [28]. As shown in the mid row of Fig. 2A, the probe pulse can stimulate fluorescence emission after the upper state $\left(\mathrm{S}_{1}\right)$ of the fluorophore is populated by the pump excitation. This process can accelerate the relaxation of the fluorophore back to the ground state $\left(\mathrm{S}_{0}\right)$ and facilitates the absorption of additional pump photons. Proper selection of the pump and probe wavelengths to match the excitation and emission peaks of the fluorophore can maximize the efficiency of stimulated emission, which results in multiple excitation-relaxation cycles within the duration of the pump pulse and thus increases the fluorophore's absorptivity of the pump photon. Moreover, increasing the pump-probe time delay reduces the stimulated emission due to the gradual depopulation of $S_{1}$. The differential optical absorption of the pump pulse in the presence and absence of the probe or due to different pump-probe delays can be utilized as a contrast mechanism for photoacoustic imaging of fluorophores with enhanced specificity.

Recently, the feasibility of stimulated emission-based photoacoustic imaging was experimentally demonstrated in a 6-mm-thick tissue phantom consisting of polymer capillaries filled with Atto680 and murine blood [28]. The pump and probe wavelengths were selected to be 680 and $742 \mathrm{~nm}$, respectively, matching the excitation and emission peaks of Atto680. As shown in Fig. 2C, the phantom was imaged with both simultaneous (i.e., non-delayed) and delayed pump-probe pulses. Subtraction of the two images completely removes the background signal from blood, which has negligible stimulated emission in this pump-probe setting, and enables high-specificity imaging of Atto680. Providing much improved spatial resolution compared to that of deep-tissue fluorescence imaging, stimulated emission-based photoacoustic imaging has potentially broad basic and translational applications. 


\section{Isotropic-resolution PAM based on ground-state depletion}

The third contrast mechanism of pump-probe photoacoustic imaging is ground-state depletion. Different from that in the excited-state absorption and stimulated emission settings, the pump and probe pulses used for photoacoustic imaging of ground-state depletion are typically of the same wavelength. In this process (bottom row of Fig. 2A), the first (i.e., pump) pulse excites the molecule from the ground state $\left(\mathrm{S}_{0}\right)$ to the upper state $\left(\mathrm{S}_{1}\right)$. Due to the pump-induced depopulation of the ground state, the second (i.e., probe) pulse experiences reduced optical absorption. The difference between the photoacoustic signals generated by the probe light in the presence and absence of the pump depends on the recovery time of $\mathrm{S}_{0}$ from depletion, providing another molecule-specific contrast mechanism for photoacoustic imaging [29].

Besides, the effect of ground-state depletion provides a way to break the conventional limit on the axial resolution of PAM [30]. In conventional PAM, the axial resolution is determined by the bandwidth of the photoacoustic signal detected by the ultrasonic transducer [2]. Refining the axial resolution to the level of the optical diffraction-limited lateral resolution requires $\mathrm{GHz}$ acoustic bandwidth, which is impractical. In ground-state depletion-based PAM, the differential signal with the pump on and off is proportional to the product of the pump and probe fluence. This nonlinear dependence offers PAM the optical-sectioning capability equivalent to that of two-photon microscopy. Indeed, with this pump-probe strategy, the axial resolution of PAM has been refined down to $1.5 \mu \mathrm{m}$-approaching the lateral resolution defined by optical focusing [31]. The isotropic resolution enables threedimensional visualization of the biconcave shape of a single RBC (Fig. 2D) [31]. It is worth noting that similar optical sectioning in PAM has also been achieved through other nonlinear processes, such as photobleaching [32] and photothermal tagging [33]. Advancing these techniques to in vivo settings will excite broad new applications.

\section{Contrast-enhanced molecular photoacoustic imaging}

Molecular photoacoustic imaging in the visible and near-infrared regions often suffers from insufficient contrast due to the substantial background generated by endogenous hemoglobin. A conventional strategy to differentiate the molecular signal of interest from the background is photoacoustic spectroscopy, which however requires repeated measurements at multiple optical wavelengths, is computationally expensive, and relies on fluence compensation to account for the spectral and spatial distortion by the tissue [34].

Recent advances in optically absorbing switchable probes have led to a promising alternative that utilizes differential detection for contrast-enhanced molecular photoacoustic imaging. One switching mechanism is magnetic field-induced motion [35]. The so-called magnetooptical probe, consisting of a magnetic core and a gold nanoshell, is both magnetically sensitive and optically absorbing. An external magnetic field can induce cyclic motion of the probe, which modulates the photoacoustic signal generated by the gold nanoshell. Motion analysis can effectively remove the magnetically insensitive background, thereby boosting the contrast by orders of magnitude over conventional photoacoustic imaging. This technique has been applied for in vivo imaging of targeted cancer cells [36]. Another switching mechanism for differential detection is photochroism [37]. BphP1—a 
nonfluorescent bacterial phytochrome-can be reversibly switched between two conformational states (i.e., Pr and Pfr) upon light excitation, resulting in a shift in its optical absorption spectrum [38]. Specifically, BphP1 undergoes the conversion from Pfr to Pr when excited with 730-790-nm light and from Pr back to Pfr upon 630-690-nm excitation.

Recently, this photochromic probe was genetically encoded in glioblastoma cells for in vivo tumor imaging [37]. As shown in Fig. 3, the mouse brain with a glioblastoma sitting at $3 \mathrm{~mm}$ beneath the scalp was repeatedly imaged at $780 \mathrm{~nm}$, during which the conformational state of the BphP1 expressed by the tumor cells was photoswitched between Pfr and Pr. In the images acquired under individual states (Fig. 3A and 3B), the tumor signal is obscured by the overwhelming vascular background. Strikingly, subtracting the two images completely removes the non-photochromic vasculature and highlights the BphP1-expressing tumor (Fig. 3C). Engineering transgenic mouse models with cell or pathway-specific expression of BphP1 will expose this high-contrast deep-penetrating molecular imaging technique to more broad applications.

\section{Summary}

Centering on the two niches of photoacoustic imaging, optical absorption contrast and spatial scalability, recent research efforts have led to rapid and exciting expansion of the technology envelope. Translating these emerging concepts into robust tools will fill important gaps in basic and translational research, including microscopic imaging of oxygen metabolism in vivo and high-resolution high-contrast molecular imaging at depth.

\section{Acknowledgments}

This work was sponsored in part by the American Heart Association National Scientist Development Grant 15SDG25960005 and the National Institutes of Health Grant EB020843.

\section{References and recommended reading}

Papers of particular interest, published within the period of review have been highlighted as:

- of special interest

1. Wang LV. Multiscale photoacoustic microscopy and computed tomography. Nat Photonics. 2009; 3:503-509. [PubMed: 20161535]

2. Wang LV, Hu S. Photoacoustic Tomography: In Vivo Imaging from Organelles to Organs. Science. 2012; 335:1458-1462. [PubMed: 22442475]

3. Zhang C, Zhang YS, Yao D-K, Xia Y, Wang LV. Label-free photoacoustic microscopy of cytochromes. J Biomed Opt. 2013; 18:20504. [PubMed: 23370407]

4. Maslov K, Zhang HF, Hu S, Wang LV. Optical-resolution photoacoustic microscopy for in vivo imaging of single capillaries. Opt Lett. 2008; 33:929-931. [PubMed: 18451942]

5. Lin L, Yao J, Li L, Wang LV. In vivo photoacoustic tomography of myoglobin oxygen saturation. J Biomed Opt. 2016; 21:61002. [PubMed: 26719943]

6. Zhang C, Maslov K, Wang LV. Subwavelength-resolution label-free photoacoustic microscopy of optical absorption in vivo. Opt Lett. 2010; 35:3195-7. [PubMed: 20890331]

7. Xu Z, Zhu Q, Wang LV. In vivo photoacoustic tomography of mouse cerebral edema induced by cold injury. J Biomed Opt. 2011; 16:066020. [PubMed: 21721821] 
8. Wang HW, Chai N, Wang P, Hu S, Dou W, Umulis D, Wang LV, Sturek M, Lucht R, Cheng JX. Label-free bond-selective imaging by listening to vibrationally excited molecules. Phys Rev Lett. 2011:106.

9. Kneipp M, Turner J, Hambauer S, Krieg SM, Lehmberg J, Lindauer U, Razansky D. Functional real-time optoacoustic imaging of middle cerebral artery occlusion in mice. PLoS One. 2014; 9:e96118. [PubMed: 24776997]

10. Heijblom M, Piras D, Brinkhuis M, van Hespen JCG, van den Engh FM, van der Schaaf M, Klaase JM, van Leeuwen TG, Steenbergen W, Manohar S. Photoacoustic image patterns of breast carcinoma and comparisons with Magnetic Resonance Imaging and vascular stained histopathology. Sci Rep. 2015; 5:11778. [PubMed: 26159440]

11. Juratli MA, Sarimollaoglu M, Siegel ER, Nedosekin DA, Galanzha EI, Suen JY, Zharov VP. Realtime monitoring of circulating tumor cell release during tumor manipulation using in vivo photoacoustic and fluorescent flow cytometry. Head Neck. 2014; 36:1207-15. [PubMed: 23913663]

12. Jansen K, van der Steen AFW, van Beusekom HMM, Oosterhuis JW, van Soest G. Intravascular photoacoustic imaging of human coronary atherosclerosis. Opt Lett. 2011; 36:597-9. [PubMed: 21368919]

13. Taruttis A, Ntziachristos V. Advances in real-time multispectral optoacoustic imaging and its applications. Nat Photonics. 2015; 9:219-227.

14. Culver JP, Ntziachristos V, Holboke MJ, Yodh AG. Optimization of optode arrangements for diffuse optical tomography: A singular-value analysis. Opt Lett. 2001; 26:701-703. [PubMed: 18040425]

15. Kim C, Favazza C, Wang LV. In vivo photoacoustic tomography of chemicals: high-resolution functional and molecular optical imaging at new depths. Chem Rev. 2010; 110:2756-82. [PubMed: 20210338]

16. Nie L, Chen X. Structural and functional photoacoustic molecular tomography aided by emerging contrast agents. Chem Soc Rev. 2014; 43:7132-70. [PubMed: 24967718]

17. Laplante M, Sabatini DM. mTOR signaling in growth control and disease. Cell. 2012; 149:274-93. [PubMed: 22500797]

18•. Yao J, Maslov KI, Zhang Y, Xia Y, Wang LV. Label-free oxygen-metabolic photoacoustic microscopy in vivo. J Biomed Opt. 2011; 16:076003. The first report on photoacoustic imaging of $\mathrm{MRO}_{2}$ in vivo. [PubMed: 21806264]

19. Yao J, Wang L, Yang J-M, Maslov KI, Wong TTW, Li L, Huang C-H, Zou J, Wang LV. High-speed label-free functional photoacoustic microscopy of mouse brain in action. Nat Methods. 2015; 12:407-410. [PubMed: 25822799]

20. Ning B, Kennedy MJ, Dixon AJ, Sun N, Cao R, Soetikno BT, Chen R, Zhou Q, Kirk Shung K, Hossack JA, et al. Simultaneous photoacoustic microscopy of microvascular anatomy, oxygen saturation, and blood flow. Opt Lett. 2015; 40:910. [PubMed: 25768144]

21 • Ning B, Sun N, Cao R, Chen R, Kirk Shung K, Hossack JA, Lee J-M, Zhou Q, Hu S. Ultrasoundaided Multi-parametric Photoacoustic Microscopy of the Mouse Brain. Sci Rep. 2015; 5:18775. The first report on simultaneous photoacoustic microscopy of absolute $\mathrm{C}_{\mathrm{Hb}}, \mathrm{sO}_{2}$ and blood flow at the microscopic level in vivo. [PubMed: 26688368]

22. Wang Y, Wang RK. Measurement of particle concentration in flow by statistical analyses of optical coherence tomography signals. Opt Lett. 2011; 36:2143-5. [PubMed: 21633476]

23. Zhou Y, Yao J, Maslov KI, Wang LV. Calibration-free absolute quantification of particle concentration by statistical analyses of photoacoustic signals in vivo. J Biomed Opt. 2014; 19:37001. [PubMed: 24589987]

24. Davydova D, de la Cadena A, Akimov D, Dietzek B. Transient absorption microscopy: advances in chemical imaging of photoinduced dynamics. Laser Photon Rev. 2016; 10:62-81.

25. Fischer MC, Wilson JW, Robles FE, Warren WS. Invited Review Article: Pump-probe microscopy. Rev Sci Instrum. 2016; 87:031101. [PubMed: 27036751]

26•. Shao Q, Morgounova E, Jiang C, Choi J, Bischof J, Ashkenazi S. In vivo photoacoustic lifetime imaging of tumor hypoxia in small animals. J Biomed Opt. 2013; 18:076019. The first report on 
excited-state absorption-based photoacoustic lifetime imaging of $\mathrm{pO}_{2}$ in vivo. [PubMed: 23877772]

27. Shao Q, Ashkenazi S. Photoacoustic lifetime imaging for direct in vivo tissue oxygen monitoring. J Biomed Opt. 2015; 20:036004. [PubMed: 25748857]

28•. Märk J, Schmitt F-J, Theiss C, Dortay H, Friedrich T, Laufer J. Photoacoustic imaging of fluorophores using pump-probe excitation. Biomed Opt Express. 2015; 6:2522-35. The first report on pump-probe photoacoustic imaging based on stimulated emission. [PubMed: 26203378]

29. Shelton RL, Mattison SP, Applegate BE. Molecular specificity in photoacoustic microscopy by time-resolved transient absorption. Opt Lett. 2014; 39:3102-5. [PubMed: 24875987]

30. Shelton RL, Applegate BE. Ultrahigh resolution photoacoustic microscopy via transient absorption. Biomed Opt Express. 2010; 1:676-686. The first report on pump-probe photoacoustic imaging based on ground-state depletion. [PubMed: 21258499]

31. Shelton RL, Mattison SP, Applegate BE. Volumetric imaging of erythrocytes using label-free multiphoton photoacoustic microscopy. J Biophotonics. 2014; 7:834-40. [PubMed: 23963621]

32. Yao J, Wang L, Li C, Zhang C, Wang LV. Photoimprint Photoacoustic Microscopy for ThreeDimensional Label-Free Subdiffraction Imaging. Phys Rev Lett. 2014; 112:014302. [PubMed: 24483902]

33. Wang L, Zhang C, Wang LV. Grueneisen relaxation photoacoustic microscopy. Phys Rev Lett. 2014; 113:174301. [PubMed: 25379919]

34. Cox B, Laufer JG, Arridge SR, Beard PC. Quantitative spectroscopic photoacoustic imaging: a review. J Biomed Opt. 2012; 17:061202. [PubMed: 22734732]

35•. Jin Y, Jia C, Huang S-W, O’Donnell M, Gao X. Multifunctional nanoparticles as coupled contrast agents. Nat Commun. 2010; 1:41. The first report on magneto-optical probe for contrastenhanced photoacoustic imaging. [PubMed: 20975706]

36. Li J, Arnal B, Wei C-W, Shang J, Nguyen T-M, O’Donnell M, Gao X. Magneto-optical nanoparticles for cyclic magnetomotive photoacoustic imaging. ACS Nano. 2015; 9:1964-76. [PubMed: 25658655]

37•. Yao J, Kaberniuk AA, Li L, Shcherbakova DM, Zhang R, Wang L, Li G, Verkhusha VV, Wang LV. Multiscale photoacoustic tomography using reversibly switchable bacterial phytochrome as a near-infrared photochromic probe. Nat Methods. 2016; 13:67-73. The first report on photochromic probe for contrast-enhanced photoacoustic imaging. [PubMed: 26550774]

38. Auldridge ME, Forest KT. Bacterial phytochromes: more than meets the light. Crit Rev Biochem Mol Biol. 2011; 46:67-88. [PubMed: 21250783] 


\section{Highlights}

- Optical absorption contrast and spatial scalability are two niches of photoacoustic imaging.

- Multi-parametric photoacoustic microscopy enables simultaneous imaging of the concentration of hemoglobin, oxygen saturation of hemoglobin, and blood flow at the microscopic level.

- $\quad$ Pump-probe photoacoustic imaging enables high-specificity molecular imaging at depth and high-resolution volumetric imaging beyond the conventional limit.

- $\quad$ Photochromic and magneto-optical probes enables high-contrast molecular photoacoustic imaging based on differential detection. 


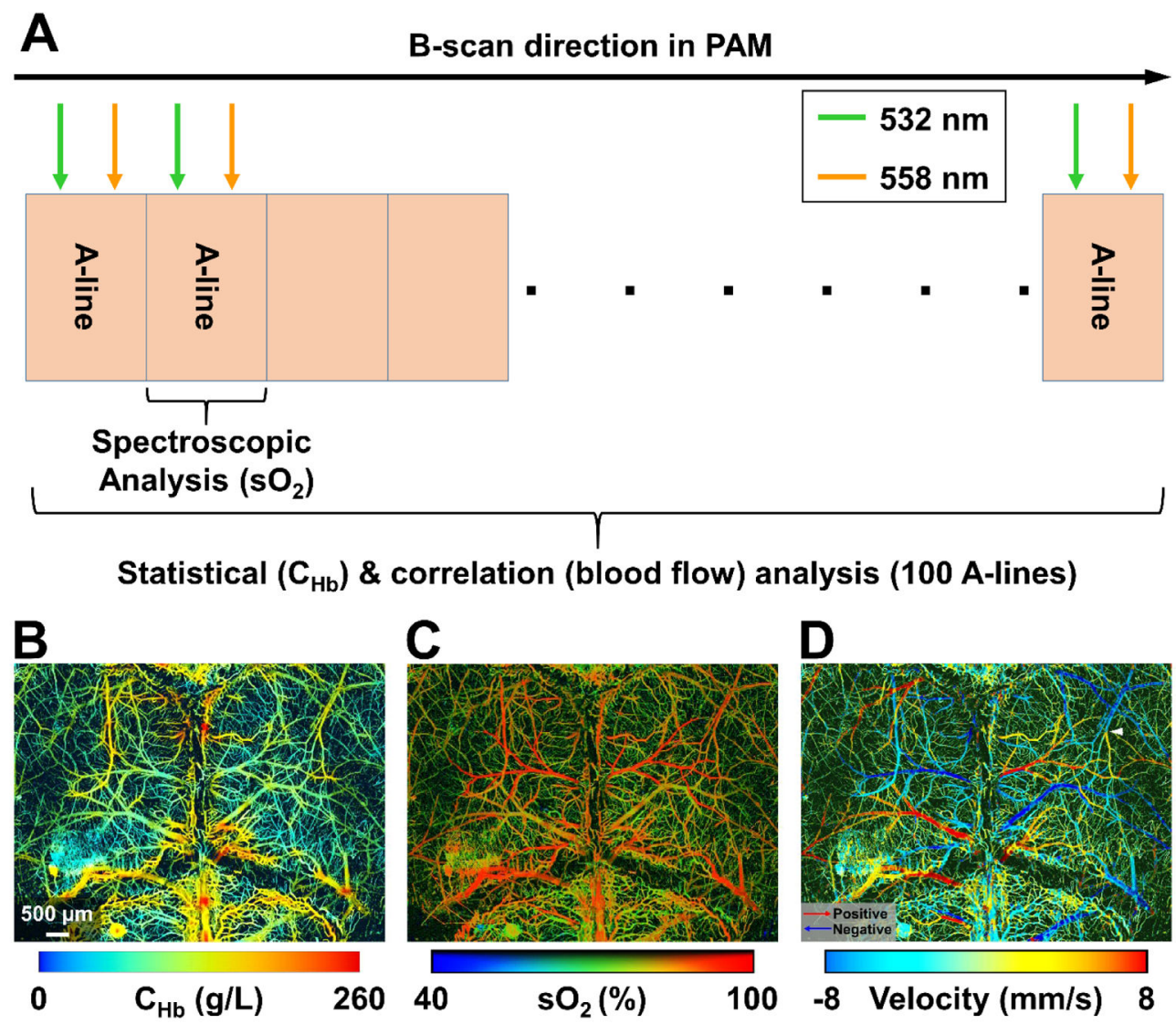

Figure 1.

Multi-parametric PAM. (A) Scanning mechanism for statistical, spectroscopic and correlation analysis. (B-D) Simultaneous PAM of $\mathrm{C}_{\mathrm{Hb}}, \mathrm{sO}_{2}$ and blood flow velocity in the mouse brain in vivo (adapted from [21]). 

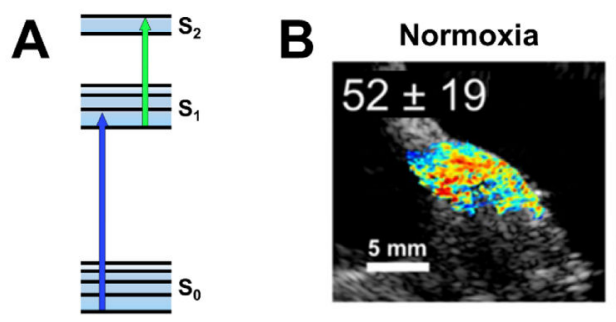

Hyperoxia

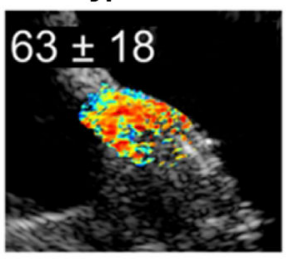

Hypoxia

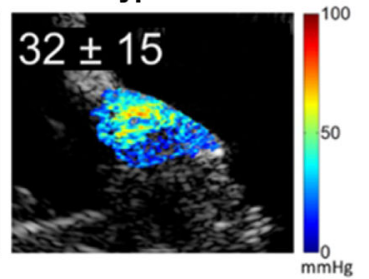

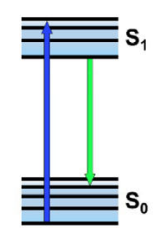

C
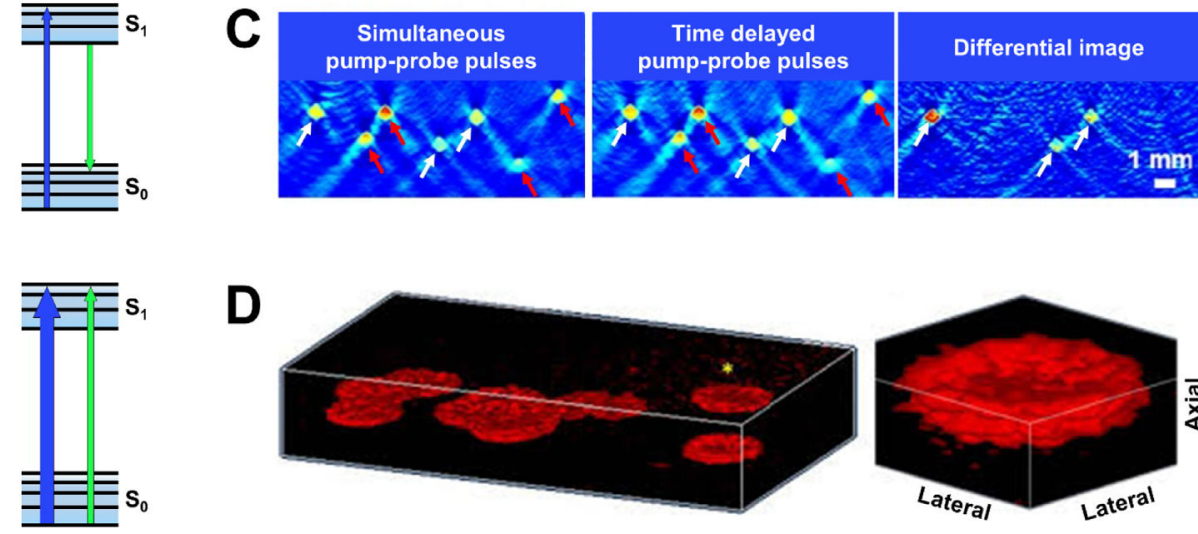

D
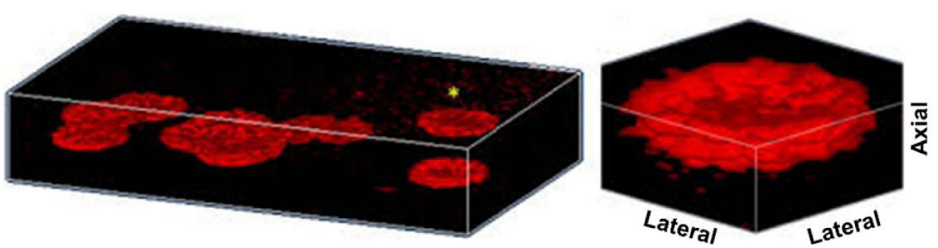

Figure 2.

Pump-probe photoacoustic imaging. (A) Simplified energy diagrams depicting excited-state absorption, stimulated emission and ground-state depletion. The blue and green arrows respectively denote the transition in the energy state due to pump and probe excitation. (B) Excited-state absorption-based photoacoustic lifetime imaging of $\mathrm{pO}_{2}$ in the mouse hindlimb under systemic normoxia $\left(21 \% \mathrm{O}_{2}\right)$, hyperoxia $\left(80 \% \mathrm{O}_{2}\right)$ and hypoxia $\left(10 \% \mathrm{O}_{2}\right)$. The concurrently acquired ultrasound image is shown in gray (adapted from [27]). (C) Stimulated emission-based photoacoustic images of a tissue phantom consisting of polymer capillaries filled with Atto680 (white arrows) and murine blood (red arrows) acquired using both simultaneous and delayed pump-probe pulses. The differential image completely eliminates the blood signals and highlights the transient absorption signal from Atto680 (adapted from [28]). (D) ground-state depletion-based isotropic-resolution PAM of fixed red blood cells in a volume of $50 \mu \mathrm{m} \times 50 \mu \mathrm{m} \times 20 \mu \mathrm{m}$. Blow-up view of a single RBC clearly shows the biconcave shape (adapted from [31]). 


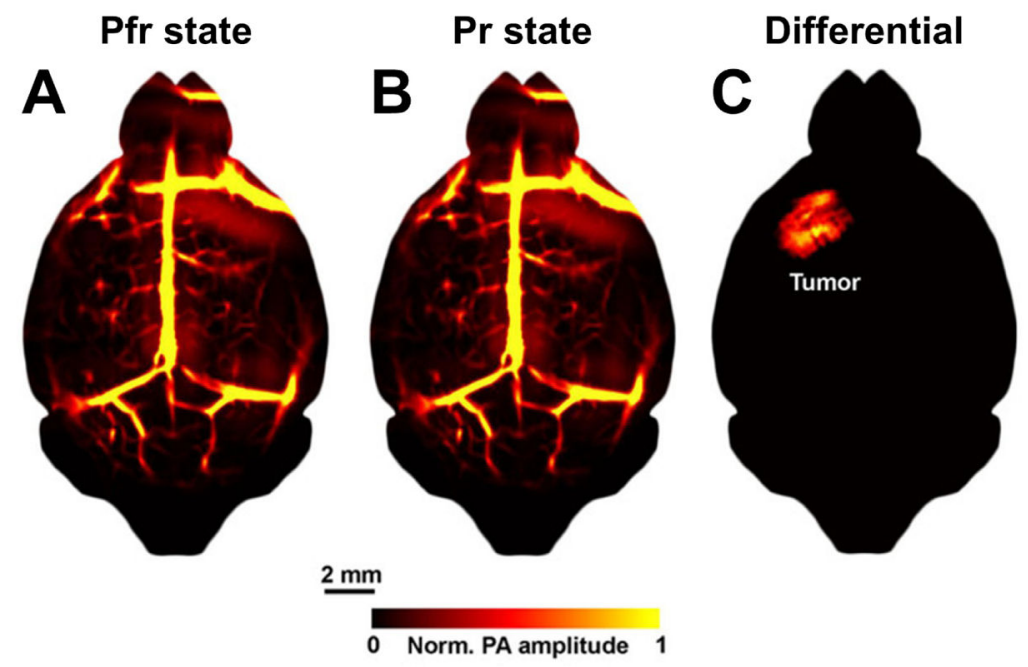

Figure 3.

Contrast-enhanced molecular photoacoustic imaging based on differential detection of photochroism. (A, B) Photoacoustic images of the tumor-bearing mouse brain acquired when the BphP1 expressed by the tumor cells was photoswitched to the Pfr state and the Pr state, respectively. The tumor is invisible in either image due to the overwhelming vascular background. (C) The differential image completely eliminates the background vasculature and clearly delineates the BphP1-expressing tumor. Images are adapted from [37]. 